\title{
Determinação analítica da performance da irrigação por sulcos abertos e em declive
}

\author{
Aristides Fraga Lima Filho*1 \\ http://dx.doi.org/10.1590/0034-737X201562030004
}

\begin{abstract}
RESUMO
Uma solução analítica foi desenvolvida, para avaliar sulcos abertos e em declive, com base na teoria de Balanço de Volume de Walker e Skogerboe (1987). Essa metodologia é capaz de obter, matematicamente, a performance de um sistema, dispensando a construção de gráficos que definem os parâmetros de avaliação. A solução matemática é baseada no ajuste de equações ao modelo potencial, no cálculo das áreas sob as curvas definidas no ajuste e na intercessão de curvas, obtendo-se, assim, a performance de sistemas de irrigação por sulcos abertos e em declive. Para validação da metodologia, foi realizado um experimento de campo, na Fazenda Experimental da Universidade Federal do Ceará, situada no município de Pentecoste, pertencente ao Centro de Ciências Agrárias. Os dados de campo foram submetidos à análise pelas duas metodologias. Os resultados mostraram que a metodologia analítica pode ser usada para avaliar a irrigação por sulcos abertos e em declive.
\end{abstract}

Palavras-chave: balanço de volume, avaliação da irrigação, irrigação superficial.

\begin{abstract}
Analytical determination of the performance of free draining, sloping furrow irrigation

An analytical solution was developed to evaluate free-draining sloping furrows based on Walker and Skogerboe (1987) volume balance approach. Its application allows the mathematical calculation of a system performance, instead of constructing graphs that define the evaluation parameters. The mathematical solution is based on fitting equations to potential models, calculating the areas under the curves defined by the fitting and on curve intersection, , from which the system performance is obtained. To validate the methodology, we conducted a field experiment at the Experimental Farm of the Federal University of Ceará, in the municipality of Pentecoste, belonging to the Center of Agricultural Sciences, where field data were analyzed by the two methods. The results showed that the analytical methodology can be used to evaluate the free draining sloping furrows irrigation.
\end{abstract}

Key words: volume balance, irrigation evaluation, surface irrigation.

\footnotetext{
Submetido em 17/05/2013 e aprovado em 18/05/2015.

${ }^{1}$ Instituto Federal de Educação, Ciência e Tecnologia da Bahia, Salvador, Bahia, Brasil. aristides@ifba.edu.br

*Autor para correspondência: aristides@ifba.edu.br
} 


\section{INTRODUÇÃO}

No Brasil, a agricultura irrigada teve um avanço significativo, com predominância dos sistemas pressurizados, em detrimento dos métodos de superfície, que são mais familiares ao homem do campo, mas em compensação, de menor eficiência.

De acordo com Walker (2003), há um grande número de fatores a serem levados em conta, quando se pensa em selecionar um sistema de irrigação. Os fatores variam em importância de local para local e de cultura para cultura, incluindo-se compatibilidade do sistema com a cultura, fatores econômicos, limitações topográficas, propriedades do solo e muitos outros fatores externos.

Segundo Mohamed et al. (2010), os projetos de irrigação por superfície são responsáveis por quase todas as áreas de terras irrigadas do mundo, sendo seu baixo desempenho objeto de estudo de muitos pesquisadores e, segundo os mesmos autores, a maioria desses projetos é construída e operada sem o uso de técnicas adequadas, com consequentes baixas uniformidade e eficiência de aplicação de água.

Dentre os sistemas de irrigação mais utilizados no Brasil, o sistema por sulcos apresenta baixa eficiência, sendo que apenas $45 \%$ da água derivada ao sulco são efetivamente utilizados pelos cultivos (Paz et al., 2000), tornando-se necessário o desenvolvimento de estratégias de manejo que permitam reduzir a quantidade de água aplicada, maximizando a sua eficiência de utilização e aumentando a lucratividade, principalmente para regiões em que a água é escassa.

Apesar dos grandes avanços tecnológicos em agricultura irrigada, por meio do desenvolvimento de modelos matemáticos que simulam o movimento da água no solo, a irrigação por sulcos apresenta, ainda, valores entre 50 e $60 \%$ de eficiência, considerados muito baixos (Carvalho et al., 2002).

Gomes \& Testezlaf (2009) citam a região de Campinas como uma das maiores produtoras de tomate de mesa, onde se irriga principalmente por sulcos, não só por tradição, mas graças aos baixos investimentos iniciais requeridos por esse sistema. Em experimento montado em uma lavoura comercial, os autores obtiveram eficiência de aplicação de água entre 25,3 e $32,2 \%$, com perdas por percolação compreendidas entre 62,3 e $74,1 \%$.

Campos \& Testezlaf (2009) avaliaram os parâmetros de desempenho da irrigação por sulcos na cultura do tomate de mesa, na região de Mogi Guaçu-SP, e encontraram eficiência de aplicação da água (EA) de 32\%, considerada muito baixa. Entretanto, Bernardo et al. (2005) afirmam que, quando adequadamente dimensionados e manejados, os sistemas de irrigação por superfície podem apresentar desempenho satisfatório e comparável com o de qualquer outro sistema.
A hidráulica dos sistemas de irrigação por gravidade tem sido estudada em um tempo relativamente curto. A computação, mediante o recurso da simulação, tem trazido muitos benefícios à agricultura irrigada, seja em termos de pesquisa, seja em práticas de campo. Hoje existem programas capazes de dimensionar e avaliar a irrigação superficial, fornecendo ao técnico condições de lidar com alternativas de manejo adequadas às situações reais.

A avaliação de sistemas de irrigação tem como objetivos determinar a eficiência do sistema, determinar quão efetivamente ele pode ser operado e melhorado e obter informações que auxiliem os engenheiros na elaboração de projetos de outros sistemas e que permitam comparar vários métodos e procedimentos operacionais, visando ao lado econômico.

Frizzone (1993) comenta que a infiltração é o fator mais importante na irrigação por superfície, pois controla a quantidade de água que entra no solo e, consequentemente, o avanço e a recessão.No entanto, a infiltração é um dos parâmetros mais difíceis de serem determinados, com precisão, no campo. Pereira (1995) cita que diversos modelos podem ser utilizados para descrever a infiltração da água e sua distribuição no perfil do solo. Ainda segundo Frizzone (1993), a infiltração é um processo complexo, dependente das propriedades físicas do solo, do seu conteúdo inicial de água, da forma de umedecimento e das variações de permeabilidade devidas ao movimento da água na superfície e ao ar retido nos poros.

Walker (1989), Lima Filho \& Souza (2009) afirmam que, em irrigação por superfície, a infiltração varia acentuadamente com a sequência das irrigações. Segundo Frizzone (1993), isto se deve às variações que ocorrem na rugosidade e na geometria da superfície em que se verifica o escoamento. Sabe-se que, para avaliar a irrigação, os parâmetros de infiltração são de fundamental importância.

Este trabalho sugere uma solução analítica para o cálculo da performance da irrigação por sulcos, com base na metodologia de Walker \& Skogerboe (1987), com a finalidade de avaliar a precisão dos resultados, facilitar a sua análise e fornecer opções de manejo adequadas às mais variadas situações, mediante o recurso da simulação.

\section{MATERIAL E MÉTODOS}

O trabalho foi desenvolvido na Fazenda Experimental do Vale do Curu, pertencente à Universidade Federal do Ceará, Brasil, localizada entre os paralelos $3^{\circ} 45^{\prime} \mathrm{e}$ $4^{\circ} 00^{\prime}$ de Latitude Sul e os meridianos $39^{\circ} 15^{\prime}$ e $39^{\circ} 30^{\prime}$ de longitude oeste, a uma altitude de 47 metros. De acordo com a classificação climática de Koppen, o clima da região é semiárido (Bswh’i), com chuvas irregulares, 
evaporação superior à precipitação, temperatura média de $18{ }^{\circ} \mathrm{C}$ e amplitude térmica média anual inferior a 5 ${ }^{\circ} \mathrm{C}$. O solo é classificado como Aluvial Eutrófico, textura franco-arenosa (Laboratório de Solos da Universidade Federal do Ceará). O comprimento dos sulcos é de 100 metros e a declividade de $0,0023 \mathrm{~m} \mathrm{~m}^{-1}$. A água de irrigação foi classificada como $\mathrm{C}_{1} \mathrm{~S}_{1}$, com CE 0,74 dS $\mathrm{m}^{-1}$ e RAS 2,03.

Objetivou-se avaliar o desempenho da irrigação por sulcos, investigando a Eficiência de aplicação de água (Ea), o Escoamento Superficial (Twr), a Eficiência de Armazenamento (Er) e a Percolação profunda (Dpr), em sulcos, ao longo de seis irrigações, tomando como base a equação de Kostiakov-Lewis modificada (equação 1):

$\mathrm{Z}=\mathrm{k} \mathrm{T}^{\mathrm{a}}+\mathrm{f}_{\mathrm{o}} \mathrm{T}$

em que:

$\mathrm{Z}=$ infiltração acumulada $\left(\mathrm{m}^{3} \mathrm{~m}^{-1}\right)$;

$\mathrm{K}$ e a = constantes empíricas;

$\mathrm{T}$ = tempo de oportunidade de infiltração (minutos);

$\mathrm{f}_{\mathrm{o}}=$ velocidade de infiltração básica $\left(\mathrm{m}^{3} \mathrm{~m}^{-1} \mathrm{~min}^{-1}\right)$.

Ao final das seis irrigações, os resultados foram submetidos à análise de variância, sendo aplicado o teste de Tukey.

\section{Irrigação preliminar}

Inicialmente, realizou-se a irrigação chamada de preliminar, sendo determinada a primeira equação de Kostiakov-Lewis modificada, para, a partir dela, fazerse o dimensionamento. Foi escolhido um sulco-teste, cerca de três metros distante dos nove sulcos que seriam utilizados no experimento. Assim, a primeira equação obtida foi a equação 2 :

$\mathrm{Z}=0,009089 \mathrm{~T}^{0,395}+0,00015 \mathrm{~T}$

Esta equação foi utilizada apenas uma vez, para o dimensionamento. Com base nela, fez-se o primeiro dimensionamento para o sulco-teste 1, aplicando-se uma lâmina de $35 \mathrm{~mm}$, com um turno de rega de sete dias, simulando uma evapotranspiração de $5 \mathrm{~mm} \mathrm{dia}^{-1}$, embora não se tenha utilizado nenhuma cultura no experimento. Esse dimensionamento foi realizado com auxílio do programa SIDIS (Rivera, 1995). A eficiência de aplicação preconizada foi de $51,38 \%$. A vazão de entrada deveria ser de $1,48 \mathrm{~L} \mathrm{~s}^{-1}$, o que não foi possível obter, por causa da ausência de uma estrutura de controle de vazão no campo experimental.

A fase de recessão, de acordo com Walker (1989), pode ser negligenciada para situações em que a declividade do sulco seja inferior a $0,0025 \mathrm{~m} \mathrm{~m}^{-1}$, o que foi observado nesta pesquisa, pois a declividade dos sulcos era de $0,0023 \mathrm{~m} \mathrm{~m}^{-1}$.
Três grupos de sulcos foram utilizados, sendo que cada grupo foi formado por um sulco teste central, ladeado por dois outros que serviram de bordadura, de forma que foram utilizados doze sulcos ao todo, três para a irrigação preliminar e nove para as demais. Cada grupo recebeu diferentes quantidades de água, de maneira que três situações foram representadas em campo: i) irrigação completa, ii) irrigação com déficit e iii) irrigação em excesso. Entre os grupos foi deixado um sulco sem água para locomoção dentro da área. Aágua era conduzida até o experimento por um canal terciário, sendo colocada em campo por sifões de $50 \mathrm{~mm}$ de diâmetro, cujas vazões de entrada e de saída foram medidas com calhas Parshall, previamente calibradas, colocadas uma no início e outra no final de cada sulco-teste (Figura 1). A água perdida por escoamento superficial era coletada por um dreno secundário e conduzida a um dreno principal.

Para o sulco-teste 1, a água era interrompida no momento exato de aplicar a lâmina requerida (irrigação completa). Para o sulco-teste 2 , o corte da água era feito quando esta atingia o final do sulco (irrigação com déficit). No sulco-teste 3 , o corte da água era feito de maneira que o tempo de oportunidade fosse o dobro do tempo dado para o sulco-teste 1 (irrigação em excesso). O experimento foi conduzido sem cultura.

A metodologia proposta por Walker \& Skogerboe (1987) define a performance do sistema de irrigação de maneira que o volume infiltrado é obtido matematicamente pela equação (3):

$V_{z}=\left(\frac{L}{2 n}\right)\left(Z_{0}+2 Z_{1}+2 Z_{2}+\cdots . .+2 Z_{n}+2 Z_{n-1}\right)$

em que:

$V_{Z}=$ volume infiltrado calculado $\left(\mathrm{m}^{3}\right)$;

$\mathrm{L}=$ comprimento do sulco $(\mathrm{m})$;

$\mathrm{n}=$ número de divisões do sulco;

$\mathrm{Z}=$ volume infiltrado por metro de sulco $\left(\mathrm{m}^{3} \mathrm{~m}^{-1}\right)$.

\section{Irrigação com déficit}

A metodologia proposta neste trabalho tem a finalidade de obter o valor de Xd (distância que define o início da área inadequadamente irrigada) de forma analítica, evitando-se a determinação gráfica. Logo, o desempenho do sistema é determinado mediante o ajuste dos dados de infiltração ao modelo potencial, do cálculo da área sob as curvas definidas pelas equações de ajuste, bem como da obtenção por interseção de curvas. $O$ parâmetro Xd só é necessário para as irrigações com déficit. Na irrigação com déficit, as plantas próximas do final do sulco não têm as suas necessidades satisfeitas, teoricamente, pois o suprimento de água foi interrompido; aplica-se uma lâmina inadequada. 
Seja a reta GE na Figura 2, descrita por:

$y=a+b x$

em que:

$\mathrm{a}=$ coeficiente linear, que é a lâmina máxima infiltrada na cabeceira do sulco $\left(\mathrm{Z}_{\max }\right)$ menos a lâmina requerida (Lreq);

$\mathrm{b}=$ coeficiente angular.

Como neste caso a declividade é praticamente nula, a equação fica:

$\mathrm{y}=\mathrm{a}$

Seja a curva Hc na Figura 2, descrita por:

$\mathrm{Z}=\mathrm{m} \cdot \mathrm{X}^{\mathrm{n}}$

em que:

$\mathrm{Z}=$ diferença entre $\mathrm{Z}_{\text {max }}$ e a lâmina infiltrada por unidade de comprimento e por unidade de espaçamento entre sulcos;

$\mathrm{X}=$ distância;

$\mathrm{L}=$ comprimento do sulco; $\mathrm{m}, \mathrm{n}=$ parâmetros de ajuste.

Seja Xd a distância que define o início da área inadequadamente irrigada. Esse ponto é determinado igualando-se as equações 4 e 6; logo o Xd é calculado pela equação:

$X d=\left(\frac{a}{m}\right)^{1 / n}$

O cálculo do volume infiltrado inadequadamente (Vzi) é obtido pela fórmula 12 conforme descrito abaixo:

O volume sob a curva entre Xd e L (comprimento do sulco) é obtido pela integral:

$V_{x d-L}=\int_{x d}^{L}\left(m \cdot X^{n}\right) d x$

$V_{x d-L}=m \int_{x d}^{L}\left(x^{n}\right) d x$

$V_{x d-L}=\left(\frac{m}{n+1}\right)\left(\left(L^{n+1}\right)-X d^{n+1}\right)$

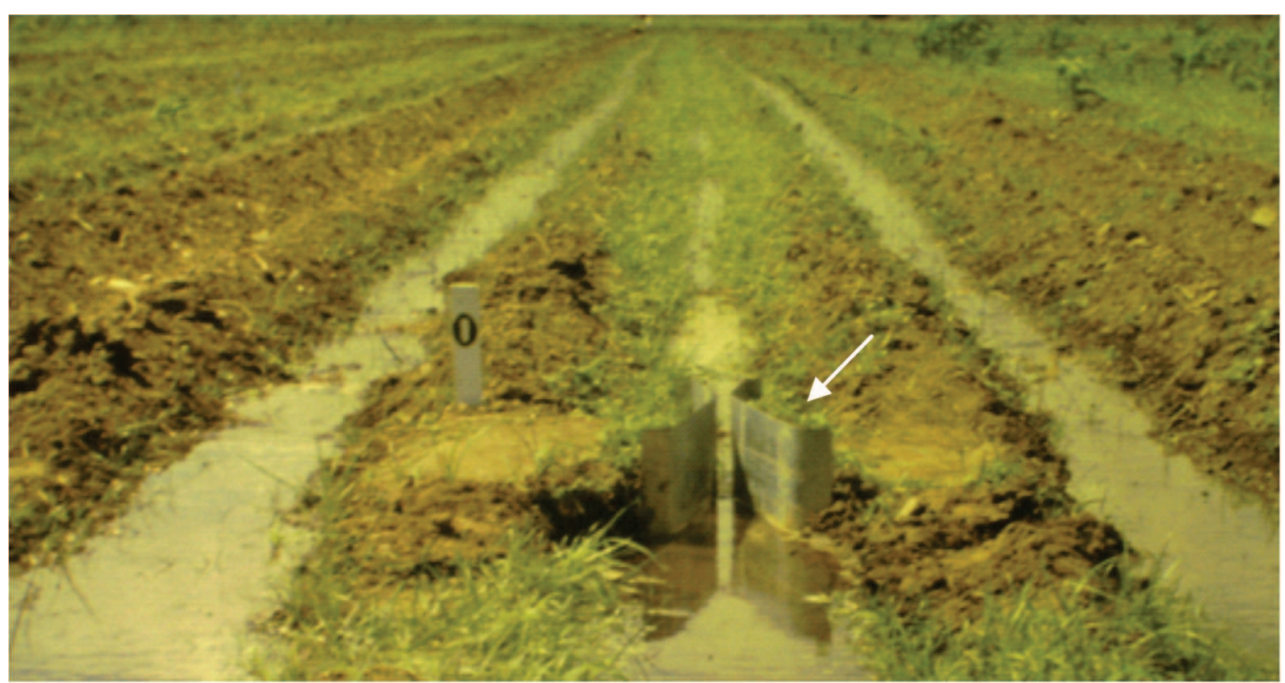

Figura 1. Calha Parshall instalada no início do sulco.

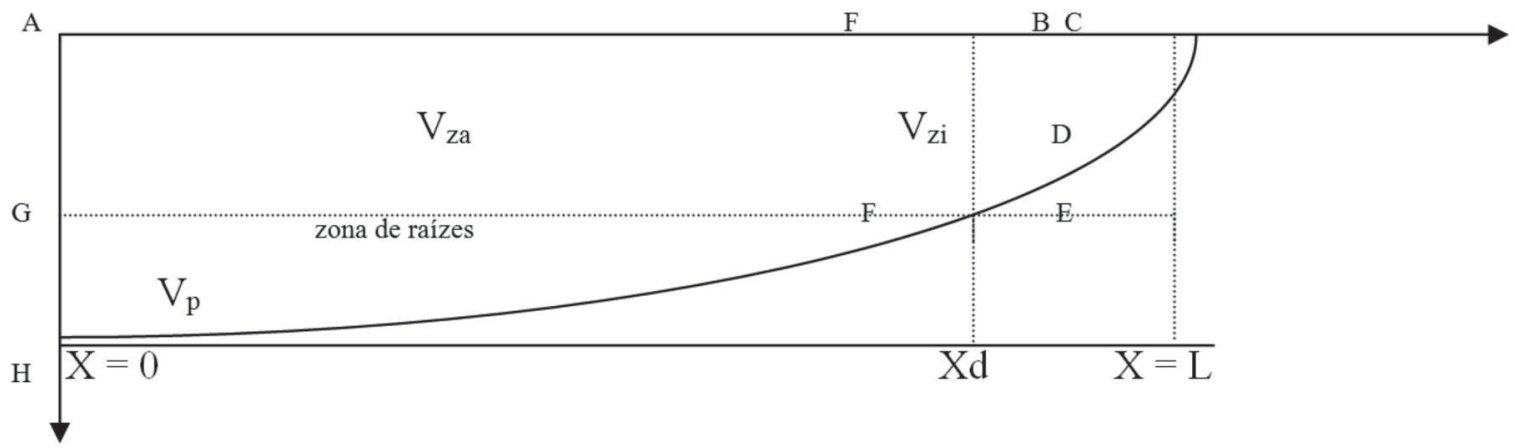

Figura 2. Lâmina infiltrada na irrigação com déficit, em que: $\mathrm{V}_{\mathrm{za}}=$ volume infiltrado adequadamente; $\mathrm{V}_{\mathrm{p}}=$ volume percolado; $\mathrm{V}_{\mathrm{zi}}=$ volume infiltrado inadequadamente; $\mathrm{Xd}=$ distância que define o início da área inadequadamente irrigada; $\mathrm{X}=$ início do sulco; $\mathrm{L}=$ final do sulco. 
O volume do retângulo F'BLXdF', da Figura 2, é calculado pela equação:

$\mathrm{V}_{\mathrm{ti}}=\mathrm{Z}_{\max } \cdot(\mathrm{L}-\mathrm{Xd}) \mathrm{Es}$

em que:

$\mathrm{V}_{\mathrm{ti}}=$ volume teórico infiltrado inadequadamente e Es espaçamento entre sulcos.

Logo, $\mathrm{V}_{\mathrm{zi}}$ é calculado pela expressão:

$V_{z i}=\left(Z_{\max }(L-X d) E s\right)-\left(\frac{m}{n+1}\right)\left(L^{n+1}-X d^{n+1}\right)$

em que

$\mathrm{V}_{\mathrm{zi}}=$ volume infiltrado inadequadamente.

$\mathrm{O}$ cálculo do volume infiltrado adequadamente $\left(\mathrm{V}_{\mathrm{za}}\right)$ é obtido pela fórmula 17 , conforme descrito abaixo:

O volume sob a área entre o início do sulco e Xd é obtido pela integral:

$V_{0-X d}=\int_{0}^{X d L}\left(m X^{n}\right) d x$

$V_{o-x d}=m \int_{0}^{X d}\left(x^{n}\right) d x$

$V_{o-X d}=\left(\frac{m}{n+1}\right)\left(X d^{n+1}\right)$

Seja o retângulo AF'XdHA, o seu volume é calculado pela equação:

$\mathrm{V}_{\mathrm{ta}}=\mathrm{Z}_{\max } \mathrm{Xd}$ Es

em que:

$\mathrm{V}_{\mathrm{ta}}=$ volume teórico infiltrado adequadamente e Es espaçamento entre sulcos.

Logo, o volume infiltrado adequadamente é calculado pela expressão:

$V_{z a}=\left(Z_{\max } X d E s\right)-\left(\left(\frac{m}{n+1}\right)\left(X d^{n+1}\right)\right)$

em que:

$\mathrm{V}_{\mathrm{za}}=$ volume infiltrado adequadamente.

As equações utilizadas para obtenção do desempemho da irrigação completa são descritas abaixo:

$$
\begin{aligned}
& E a=\frac{Z \cdot(X d+V z i)}{Q_{0} \cdot T_{c o}} \\
& D p r=\frac{V z a-(Z \cdot X d)}{Q_{0} \cdot T_{C O}} \\
& T w r=100-E a-D p r \\
& \mathrm{Er}=\frac{(Z \cdot X d)+V z i}{Z \cdot L}
\end{aligned}
$$

\section{Irrigação completa}

$\mathrm{Na}$ irrigação completa não existe déficit na zona radicular, ficando todas as plantas com suas necessi- dades satisfeitas, pois a lâmina aplicada corresponde à profundidade do sistema radicular. No início do sulco, ocorre certa perda por percolação, dada a necessidade de se aplicar uma lâmina adequada a todas as plantas.

A equação 22 é utilizada para integrar o volume sob a área entre $\mathrm{X}=0$ e $\mathrm{X}=\mathrm{L}$ :

$V_{0-L}=\int_{0}^{L}\left(m X^{n}\right) d x$

$V_{0-L}=m \cdot \int_{0}^{L}\left(X^{n}\right) d x$

$V_{0-L}=\left(\frac{m}{n+1}\right)\left(X^{n+1}\right)$

$V_{0-L}=\left(\frac{m}{n+1}\right)\left(L^{n+1}\right)$

O volume total, área ABLEA na figura seguinte é definido pela expressão:

$\mathrm{V}_{\mathrm{t}}=\mathrm{Z}_{\text {max }}$ Es L

O volume infiltrado, representado pela Figura 3, é calculado pela diferença entre o volume total e o volume da área sob a curva, já definido.

As equações utilizadas para obtenção do desempenho da irrigação completa são descritas abaixo:

$E a=\frac{Z L}{Q_{0} T_{c o}}$

$D p r=\frac{V z-(z L)}{Q_{O} T_{C O}}$

$T w r=100-E a-D p r$

$\mathrm{Er}=100 \%$,

em que:

$\mathrm{Z}=$ volume infiltrado por unidade de comprimento e por unidade de largura $\left(\mathrm{m}^{3} \mathrm{~m}^{-1}\right)$;

$\mathrm{Q}_{\mathrm{o}}=$ vazão de entrada $\left(\mathrm{L} \mathrm{s}^{-1}\right)$;

$\mathrm{T}_{\text {co }}=$ tempo de corte $(\mathrm{min})$;

$\mathrm{Vz}=$ volume infiltrado;

Ea = eficiência de aplicação;

Dpr = percolação profunda;

Twr = taxa de escoamento superficial;

$\mathrm{Er}=$ eficiência de armazenamento.

\section{Irrigação em excesso}

$\mathrm{Na}$ irrigação em excesso, ocorre perda de água desnecessária, pois a zona de raízes está suficientemente abastecida, por aplicação de lâmina maior que a requerida pelas culturas, e isto é o que ocorre muitas vezes nos perímetros irrigados. 
O volume infiltrado é calculado com a equação 22, integrando-se o volume sob a área entre $\mathrm{X}=0$ e $\mathrm{X}=\mathrm{L}$, conforme visto anteriormente.

$\mathrm{O}$ volume infiltrado, representado pela área ABEGA, da Figura 4, é calculado pela diferença entre o volume total, dado por ABLGA, e o volume sob a curva 22:

$\mathrm{V}_{\mathrm{z}}=\mathrm{V}_{\mathrm{t}}-\mathrm{V}_{0-\mathrm{L}}$

em que:

$\mathrm{V}_{\mathrm{z}}=$ volume infiltrado;

$\mathrm{V}_{\mathrm{t}}=$ volume total;

$\mathrm{V}_{0-\mathrm{L}}=$ volume sob a curva 22 .

As equações utilizadas para obtenção do desempenho da irrigação em excesso são descritas abaixo:

$$
\begin{aligned}
& E a=\frac{Z L}{Q_{0} T_{c o}} \\
& D p r=\frac{V z-(Z L)}{Q_{O} T_{C O}} \\
& T w r=100-E a-D p r \\
& \mathrm{Er}=100 \% .
\end{aligned}
$$

\section{RESULTADOS E DISCUSSÃO}

A Tabela 1 apresenta os resultados encontrados para os parâmetros de avaliação das irrigações, segundo as duas metodologias empregadas, e nela se percebe a boa aproximação entre os dados obtidos pela determinação analítica e os dados calculados pela metodologia de Walker \& Skogerboe (1987). Os resultados obtidos com as metodologias testadas apresentaram diferenças que variaram de 0 a 16,3\%, com média de $5,5 \%$, considerando-se os resultados das seis irrigações, que foram submetidos à análise de variância e não apresentaram diferenças significativas, a $1 \%$, pelo teste de Tukey.

Observam-se no sulco 2 as maiores taxas de eficiência de aplicação de água (Ea), com média de 58,3\% para as seis irrigações, por causa de o corte da água ter sido feito com a intenção de provocar o déficit hídrico, melhorando-se, assim, a eficiência de aplicação de água, enquanto, no sulco 3, percebem-se as mais baixas, com média de $34,07 \%$, por causa de o tempo de oportunidade de infiltração ter sido o dobro daquele aplicado ao sulco 1. Para o sulco 1, observa-se que os resultados foram relativamente altos, próximos dos resultados do sulco 2, por causa de o corte da água ter sido feito para atender à irrigação completa, com média de 46,16\% de eficiência de aplicação de água. Estes resultados estão na faixa normalmente encontrada em irrigação por sulcos, que, segundo Smith et al. (2005), encontra-se em torno de $45 \%$. De acordo com Bernardo et al. (2005), esses valores são considerados baixos, pois no mínimo deveriam ser de $60 \%$.

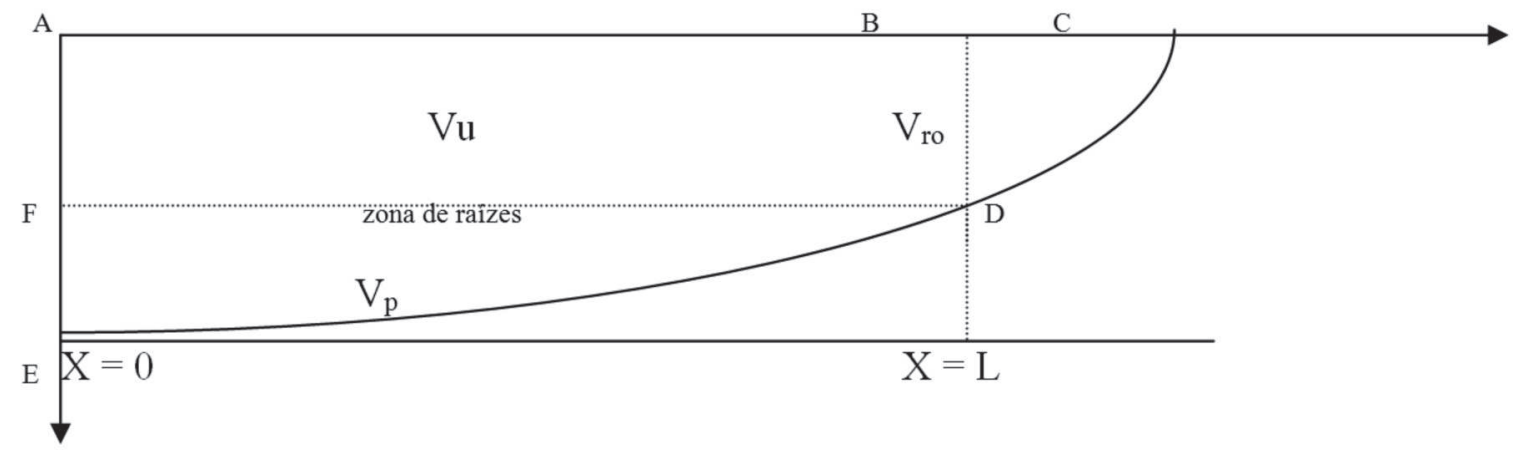

Figura 3. Lâmina infiltrada da irrigação completa, em que: $\mathrm{Vu}=$ volume útil; $\mathrm{V}_{\mathrm{p}}=$ volume percolado; $\mathrm{V}_{\mathrm{ro}}=$ volume de run off; $\mathrm{X}=$ início do sulco; $\mathrm{L}=$ final do sulco.

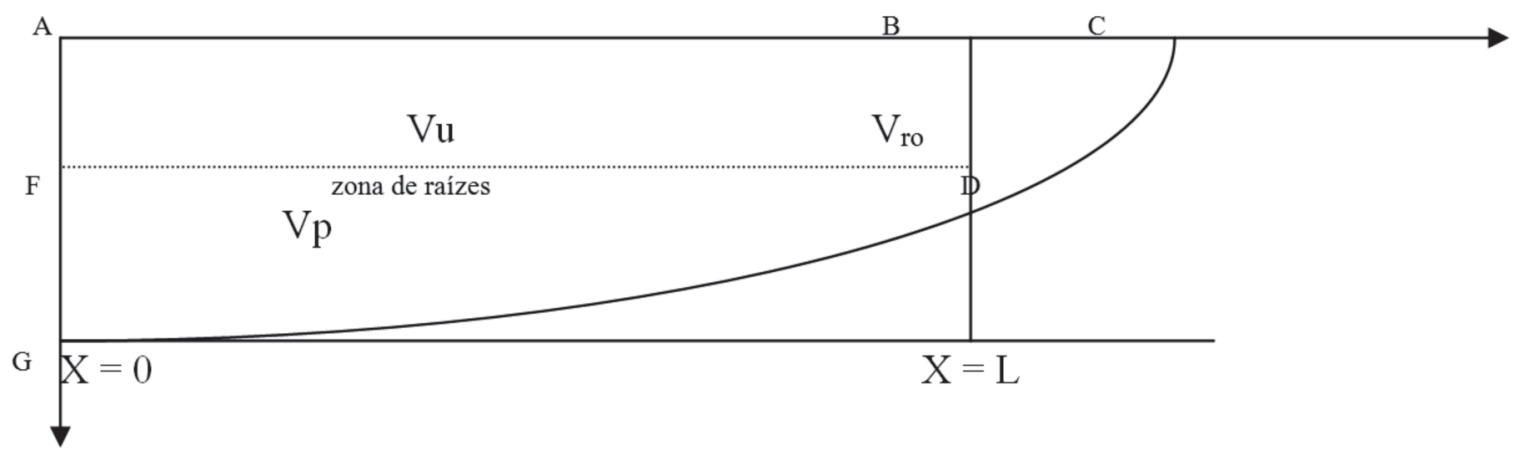

Figura 4. Lâmina infiltrada na irrigação em excesso, em que: $\mathrm{Vu}=$ volume útil; $\mathrm{V}_{\mathrm{p}}=$ volume percolado; $\mathrm{V}_{\mathrm{ro}}=$ volume de run off; $\mathrm{X}=$ início do sulco; $\mathrm{L}=$ final do sulco. 
Resultados semelhantes foram obtidos por Castro (1997), analisando a irrigação por sulcos em cultura de feijão de corda, utilizando a metodologia do balanço de volume de Walker \& Skogerboe (1987), que encontrou eficiência de aplicação variando de 40,8 a $74,5 \%$. Já Gomes \& Testezlaf (2009) encontraram eficiência de aplicação entre 25,3 e 32,2\%, em cultivo comercial de tomate, na região de Campinas-SP.

Os sulcos utilizados neste trabalho foram abertos no final, proporcionando eficiências mais baixas. Essa água escoada no final do sulco poderia ser reaproveitada por meio de bombeamento; porém, há que se considerar o fator econômico, em virtude dos gastos de energia e de mão de obra. Portanto, a questão não se resume em aumentar a eficiência de um lado, esquecendo-se a relação benefício/custo, de outro. Por outro lado, Scaloppi (2003) afirma que sulcos fechados em nível têm potencial para alcançar valores de eficiência de aplicação acima de $80 \%$, compatíveis com os de sistemas pressurizados. Para a taxa de escoamento superficial (Twr), por causa de os sulcos serem abertos e em declive, os resultados foram coerentes com o tempo de corte da água, sendo média de 50,4\%, para o sulco 1 de irrigação com- pleta, de 40,7\%, para o sulco 2 de irrigação com déficit e de $61,4 \%$, para o sulco 3, com maior desperdício de água, por excesso de aplicação. No sulco 3, observamse os maiores valores para o escoamento superficial, dada a maior quantidade de água aplicada.

Com relação à eficiência de armazenamento de água (Er), a média para o sulco 1 foi de $84,4 \%$, de $61,7 \%$ para o sulco 2 e de $95,7 \%$ para o sulco 3. Esses valores não atingiram 100\%; porém, segundo Frizzone (1999), valores muito baixos encontrados em campo indicam ineficiência do sistema e são indesejáveis.

A percolação profunda (Dpr) foi pequena em todos os sulcos, talvez porque o solo trabalhado, apesar de sua textura franco-arenosa, comportou-se como um solo com maiores teores de argila. Assim, observa-se que na primeira irrigação as perdas foram de 12,9; 6,2 e 17,4\%, respectivamente, para os sulcos 1,2 e 3 , enquanto para as demais irrigações as perdas foram praticamente nulas, sugerindo que este solo oferece dificuldades para a infiltração da água, diferentemente de Gomes \& Testezlaf (2009) que obtiveram valores de perdas por percolação compreendidos entre 62,3 e 74,1\%, em solo Argissolo Vermelho-Amarelo, de textura franco-arenosa.

Tabela 1. Eficiência de aplicação da água (Ea), Taxa de Escoamento Superficial (Twr), Eficiência de Armazenamento (Er) e Percolação profunda (Dpr), para os três sulcos de irrigação ao longo das seis irrigações na Fazenda Experimental do Vale do Curu, pertencente à Universidade Federal do Ceará, Brasil

\begin{tabular}{|c|c|c|c|c|c|c|c|}
\hline \multirow{2}{*}{ Irrigação } & \multirow{2}{*}{ Parâmetro } & \multicolumn{2}{|l|}{ Sulco 1} & \multicolumn{2}{|l|}{ Sulco 2} & \multicolumn{2}{|l|}{ Sulco 3} \\
\hline & & Walker/Sko & Det. Analítica & Walker/Sko & Det. Analítica & Walker/Sko & Det. Analítica \\
\hline \multirow{4}{*}{$1^{\mathrm{a}}$} & $\mathrm{Ea}$ & 54,77 & 60,74 & 58,49 & 68,05 & 53,95 & 53,00 \\
\hline & Twr & 26,38 & 23,33 & 27,84 & 25,75 & 30,07 & 28,69 \\
\hline & $\mathrm{Er}$ & 89,17 & 98,90 & 80,99 & 94,44 & 100,00 & 100,00 \\
\hline & Dpr & 18,85 & 12,93 & 13,67 & 06,21 & 15,98 & 17,36 \\
\hline \multirow{4}{*}{$2^{\mathrm{a}}$} & $\mathrm{Ea}$ & 54,49 & 54,53 & 46,19 & 62,53 & 42,57 & 47,56 \\
\hline & Twr & 45,51 & 45,47 & 53,81 & 37,47 & 52,01 & 51,99 \\
\hline & $\mathrm{Er}$ & 81,59 & 81,54 & 44,57 & 60,36 & 85,55 & 95,58 \\
\hline & Dpr & 00,00 & 00,00 & 00,00 & 00,00 & 05,42 & 00,45 \\
\hline \multirow{4}{*}{$3^{\mathrm{a}}$} & $\mathrm{Ea}$ & 44,78 & 44,79 & 59,52 & 62,24 & 32,95 & 32,00 \\
\hline & Twr & 55,22 & 55,21 & 40,48 & 37,76 & 67,05 & 67,05 \\
\hline & $\mathrm{Er}$ & 71,16 & 71,18 & 47,18 & 49,34 & 88,39 & 89,00 \\
\hline & Dpr & 00,00 & 00,00 & 00,00 & 00,00 & 00,00 & 00,00 \\
\hline \multirow{4}{*}{$4^{\mathrm{a}}$} & $\mathrm{Ea}$ & 29,10 & 29,00 & 54,34 & 55,19 & 15,15 & 15,31 \\
\hline & Twr & 70,90 & 70,00 & 45,66 & 44,81 & 84,55 & 84,40 \\
\hline & $\mathrm{Er}$ & 80,28 & 80,29 & 43,17 & 43,85 & 100,00 & 100,00 \\
\hline & Dpr & 00,00 & 00,00 & 00,00 & 00,00 & 00,29 & 00,29 \\
\hline \multirow{4}{*}{$5^{\mathrm{a}}$} & $\mathrm{Ea}$ & 51,43 & 51,39 & 52,76 & 57,02 & 25,35 & 32,54 \\
\hline & Twr & 44,75 & 44,76 & 47,24 & 42,98 & 66,25 & 61,45 \\
\hline & $\mathrm{Er}$ & 99,84 & 99,81 & 68,60 & 69,55 & 100,00 & 100,00 \\
\hline & Dpr & 03,82 & 03,85 & 00,00 & 00,00 & 08,40 & 06,01 \\
\hline \multirow{4}{*}{$6^{\mathrm{a}}$} & $\mathrm{Ea}$ & 36,51 & 36,52 & 43,74 & 44,58 & 24,43 & 24,00 \\
\hline & Twr & 63,49 & 63,48 & 56,21 & 55,42 & 75,57 & 75,00 \\
\hline & $\mathrm{Er}$ & 74,91 & 74,91 & 51,69 & 52,61 & 89,75 & 89,75 \\
\hline & Dpr & 00,00 & 00,00 & 00,00 & 00,00 & 00,00 & 00,00 \\
\hline
\end{tabular}


Na Tabela 2, observa-se a variação dos parâmetros a, k e f da equação de Kostiakov-Lewis, obtidos nas seis irrigações. Observa-se que o parâmetro "a" apresentou tendência a aumentar ao longo das irrigações, embora tenha atingido um valor maior na quarta irrigação. Castro \& Souza (1999) também observaram essa tendência. Os autores citam a complexidade do processo de infiltração relatada na literatura, em relação à qual as propriedades físicas do solo, o seu conteúdo de água, as variações de rugosidade e de geometria do sulco influenciam na infiltração da água.

Sabe-se também que o solo em estudo é Aluvial eutrófico, de textura franco-arenosa e com grande variabilidade espacial pela sua própria estrutura. A frequência de irrigação de sete dias foi tempo suficiente para se obsevarem fendas no solo, que eram fechadas durante o avanço da água, o que diminui a sua capacidade de infiltração, influenciando possivelmente na performance da irrigação.

Percebe-se um decréscimo dos valores do parâmetro "k", como obtido por Castro \& Souza (1999), assim como se observa um decréscimo dos valores da velocidade de infiltração básica $\left(\mathrm{f}_{\mathrm{o}}\right)$, uma vez que houve formação de um selamento superficial, pois foi observado, no decorrer das irrigações, que muitas partículas foram arrastadas por ação erosiva da água. A variação de "f $f_{o}$ " foi mais acentuada entre a primeira e a segunda irrigações, sendo que a partir da terceira irrigação os valores foram mais próximos, com tendência à estabilização. Este decréscimo de "fo", segundo Castro \& Souza (1999), ocorre por causa do processo de carreamento das partículas mais finas de um local para o outro, preenchendo os espaços porosos e diminuído a infiltração da água no solo.

\section{CONCLUSÕES}

A determinação analítica da irrigação por sulcos aproximou-se daquela obtida por Walker \& Skogerboe (1987) e pode ser usada para avaliar a irrigação por sulcos abertos e em declive. A formação de selamento su-

Tabela 2. Parâmetros da equação de Kostiakov-Lewis, nas seis irrigações realizadas na Fazenda Experimental do Vale do Curu, pertencente à Universidade Federal do Ceará, Brasil

\begin{tabular}{lccc}
\hline Irrigações & $\mathbf{a}$ & $\begin{array}{c}\mathbf{K} \\
\left(\mathbf{m}^{3} \mathbf{m i n}^{\mathbf{a}} \mathbf{m}^{-1}\right)\end{array}$ & $\begin{array}{c}\mathbf{f}_{\mathbf{o}} \\
\left(\mathbf{m}^{3} \mathbf{m i n}^{-\mathbf{1}} \mathbf{m}^{-1}\right)\end{array}$ \\
\hline 1 & 0,02936 & 0,02155 & 0,000227 \\
2 & 0,02066 & 0,00956 & 0,000080 \\
3 & 0,17126 & 0,00817 & 0,000031 \\
4 & 0,20766 & 0,00692 & 0,000010 \\
5 & 0,16324 & 0,00691 & 0,000042 \\
6 & 0,15534 & 0,00546 & 0,000019 \\
\hline
\end{tabular}

perficial certamente foi responsável pelas modificações da infiltração da água ao longo das irrigações, consequentemente alterando os parâmetros da equação de Kostiakov-Lewis e influenciando o desempenho do sistema.

\section{REFERÊNCIAS}

Bernardo S, Soares AA \& Mantovani EC (2005) Manual de Irrigação. 7a ed. Viçosa, Universidade Federal de Viçosa. 611p.

Campos MA \& Testezlaf R (2009) Simulação da eficiência e da redução do consumo de água na produção do tomate de mesa sob irrigação por sulcos. Engenharia na agricultura, 17:375-382.

Carvalho JM de, Vieira RCS \& Daniel L (2002) Determinação das características de avanço da água em sulcos de irrigação. In: Congresso Brasileiro de Engenharia Agrícola, Salvador. Anais, SBEA. CD-ROM.

Castro LCA (1997) Análise da irrigação por sulcos utilizando a metodologia do balanço de volume. Dissertação de Mestrado. Universidade Federal do Ceará, Fortaleza. 91p.

Castro LCA \& Souza F (1999) Análise dos parâmetros da equação de infiltração de Kostiakov-Lewis na irrigação por sulcos. Revista Brasileira de Engenharia Agrícola e Ambiental, 3:167-172.

Frizzone JA (1999) Anotações de aula: avaliação do desempenho de sistemas de irrigação. São Paulo, Escola Superior de Agricultura "Luiz de Queiroz". 14p.

Frizzone JA (1993) Irrigação por superfície. São Paulo, ESALQ. 183p.

Gomes EP \& Testezlaf R (2009) Desempenho da irrigação por sulcos na tomaticultura-de-mesa da região de Campinas-SP. Irriga, 14:289-298.

Lima Filho AF \& Souza F (2009) Variação temporal dos parâmetros da equação de infiltração de Kostiakov-Lewis na irrigação por sulcos. In: XIX Congresso Nacional de Irrigação e Drenagem, Montes Claros. Anais, CONIRD. 245p.

Mohamed HI, Abas OM, Elabdin Araz EL \& Ramlawi HR (2010) Development of a Muskingum- Cunge routing model for design of furrow irrigation. Agriculture And Biology Journal of North America, 1:1014-1030.

Paz VPS, Teodoro REF \& Mendonça FC (2000) Recursos hídricos, agricultura irrigada e meio ambiente. Revista Brasileira de Engenharia Agrícola e Ambiental, 4:465-473.

Pereira LS (1995) Surface irrigation system. In: Pereira LS (Ed.) Sustentainability of water resources in agriculture. Dordrecht, Kluwer. p.269-289.

Rivera RNC (1995) Desenvolvimento de um software para Dimensionamento de sistemas de irrigação por sulcos via balanço de volume. Dissertação de Mestrado. Universidade Federal do Ceará, Ceará. $1110 \mathrm{p}$.

Scaloppi EJ (2003) Sistemas de irrigação por superfície. In: Miranda JH \& Pires RCM (Eds.) Série engenharia agrícola: irrigação. Jaboticabal, FUNEP. p.311-404.

Smith RJ, Raine SR \& Minkevich J (2005) Irrigation application efficiency and deep drainage potential under surface irrigated cotton. Agricultural Water Management, 71:117-130.

Walker WR \& Skogerboe GV (1987) Surface irrigation: theory and practice. Englewood Cliffs, Prentice Hall. 386p.

Walker WR (1989) Guidelines for designing and evaluation surface irrigations systems. Rome, FAO. 137p.

Walker WR (2003) SIRMOD III - Surface Irrigation Simulation, Evaluation and Design (Guide and Technical Documentation). Logan, Utah State University. 130p.

Rev. Ceres, Viçosa, v. 62, n.3, p. 251-258, mai-jun, 2015 